\title{
AN AIRSPACE ANALYSIS ACCORDING TO AREA NAVIGATION REQUIREMENTS
}

\author{
Ivan OSTROUMOV (D)*, Volodymur KHARCHENKO (D), Nataliia KUZMENKO (ㄷ) \\ Air Navigation Systems Department, National Aviation University, Kyiv, Ukraine \\ Received 28 January 2019; accepted 29 April 2019
}

\begin{abstract}
Aircraft navigation within controlled airspace is carried out using on-board positioning systems capable to determine the coordinates of aircraft location with the system performance that meet navigation specifications requirements. The article proposes a descriptive set theory use to navigational aids network analysis in order to determine the positioning performance of the navigation system at predefined airspace volume. The uniqueness of the study is shape evaluation of areas that correspond to navigation specifications requirements and area research of different positioning techniques based on navigational aids such as DME/DME, VOR/DME, and VOR/VOR. An analysis of Ukrainian airspace has been done as an example.
\end{abstract}

Keywords: navigation, RNAV, PBN, navigational aids, performance, area, air space, aircraft, DME, VOR.

\section{Introduction}

Aircraft flights in controlled airspace are performed with the guarantee of air transport safety. The risk of dangerous events occurrence is considered as a safety criterion in civil aviation (International Civil Aviation Organization [ICAO], 2008a). Particular attention during piloting is given for aircraft navigation in the airspace. The accuracy of planned flight trajectory maintaining by each airspace user directly affects air traffic safety. In accordance with the Performance-based Navigation ( $\mathrm{PBN}$ ), positioning system characteristics are represented by accuracy, integrity, continuity, and availability (ICAO, 2008b).

Accuracy is a difference between the estimated and actual aircraft position. Integrity usually is defined as a measure of trust which can be placed in the correctness of the information supplied by the total system. Continuity is represented by the capability of the system to perform its function without unscheduled interruptions during the intended operation. Availability is characterized by a portion of time during which the system is simultaneously delivering the required level of accuracy, integrity, and continuity (ICAO, 2006). Values of these characteristics are defined in PBN specification that is implemented in each part of airspace and specify the precision of an aircraft trajectory maintaining. It should be noted that integrity, continuity, and availability are probabilistic values that more closely describe the ability of the positioning system to perform its functions within the planned characteristics, respectively, depending on the value of each of the components. Nowadays, different methods of aircraft position detection are available for airspace users. Global Navigation Satellite System (GNSS) is the most commonly used positioning system because, in comparison with other systems, it can guarantee the high level of accuracy and availability regardless aircraft position in airspace (ICAO, 2012). However, the effect of factors such as ionospheric delays (Dabbakuti, Ratnam, \& Sunda, 2016), tropospheric errors (Kutsenko, Ilnytska, \& Konin, 2018), the interference of radio waves or an unintentional jamming of signals reduces the positioning characteristics in a particular limited area (Narins et al., 2012; Lubbers, Mildner, Onincx, \& Scheele, 2015) or may cause a positioning system lock (Kasperovych, Shvets, \& Ostrovsky, 2008). Inertial Navigational System or positioning by pairs of navigational aids are used as a stand-by positioning technique in case of GNSS malfunction (Han, Gong, Meng, Li, \& Gu, 2016).

The time frame of inertial navigation systems use is limited due to the continuous increase of error. An algorithms of positioning by navigational aids, operating in the Flight Management System (FMS) computer, use data from ground angular or/and distance measuring equipment to estimate an aircraft location (Han et al., 2016; Kuzmenko, Ostroumov, \& Marais, 2018; Vitan, Berz, Saini, Arethens, Belabbas, \& Hotmar, 2018). Typical algorithm is based on

*Corresponding author. E-mail: ostroumovv@ukr.net 
data from only one pair of navigational aids, which are determined by a certain criterion of optimality (Ostroumov, Kuzmenko, \& Marais, 2018). Localization methods such as TOA (Time of arrival), AOA (Angle of arrival) or AOA/ TOA may be used for positioning by navigational aids. In case of TOA, the distances are measured by Distance Measuring Equipment (DME) and VHF omnidirectional range (VOR) or Automatic Direction Finder (ADF) can be used as angular data sensors for AOA method. The DME/DME and VOR/DME pairs are the most frequently used, while VOR/VOR and ADF/ADF are extremely rarely used due to significant errors of angle measurement by VOR and ADF equipment (Kuzmenko et al., 2018).

Onboard positioning system should meet numerous requirements for the characteristics of its operation as set in the RNP/RNAV navigation specifications. Numerous advantages of RNP/RNAV procedures makes them useful in the current airspace structure (Muller, Uday, \& Marais, 2011). Therefore, each positioning method corresponds to a certain airspace volume within which the requirements of navigational specifications approved by the Air Navigation Service Provider (ANSP) for defined airspace are met. An airspace air navigation service analysis take a significant intention at SESAR project due to segmentation of ground navigational aids within European volume into the parts with particular boundaries and characterized by different performance level. The assessment task of the airspace area compliance with the requirements of area navigation is an important stage in the assessment improvement of flight safety (Eurocontrol, 2008).

\section{Performance requirements}

The accuracy, integrity and continuity requirements are set forward up to location data obtained from the onboard positioning system. The accuracy of navigational measure- ments is characterized by Total System Error (TSE), which must take a value within the permitted levels, depending on the type of navigation specification (Table 1), in 95\% of the total flight time with longitudinal and lateral deviations (ICAO, 2008b).

According to (ICAO, 2008b), the main components of the TSE are the Navigation System Error (NSE), Flight Technical Error (FTE), and Path Definition Error (PDE):

$$
T S E^{2}=N S E^{2}+F T E^{2}+P D E^{2} .
$$

The NSE value is estimated by the maximum value of mean squared deviation error of aircraft location detection within the 95\% confidence band. NSE value depends on the type of navigation system and depends on the geometry of the ground stations location.

FTE characterizes the ability of a pilot or an automatic piloting system to follow a predetermined trajectory of flight. In the case of manual control, the FTE includes the indication or data interpretation errors. The FTE value for the various flight phases are shown in Table 2 (ICAO, 2008b; Lo et al., 2010)

The PDE includes errors of aircraft trajectory settings in the FMS and other related components of the errors (database errors, air navigation chart errors, etc.). However, due to the usage of digital databases in FMS, the PDE is too small in comparison with the value of other components and it can be neglected.

The integrity of the navigation information is defined by the value of the probability of significant failure in the positioning equipped, which, according to (ICAO, 2008b), can not exceed $10^{-5}$ per flight hour independently from flight phase. The requirements for the availability of ground navigational aids infrastructure or the segment of satellites for GNSS are also considered as a probability of a significant fault, which, according to (ICAO, 2008b), can not exceed $10^{-7}$.

Table 1. Performance requirements of navigation specification

\begin{tabular}{|l|c|c|c|}
\hline \multicolumn{1}{|c|}{ Navigation specification } & TSE, NM & Integrity & Risk of signal-in-space error \\
\hline RNAV 10 & 10 & $10^{-5}$ & $10^{-7}$ \\
\cline { 1 - 2 } RNAV 5 & 5 & \\
RNP 4 & 4 & \\
RNAV 2, RNP 2 & 2 & \\
\cline { 1 - 2 } RNAV1, RNP 1 & 1 & \\
\hline
\end{tabular}

Table 2. FTE values according to the flight phase

\begin{tabular}{|l|c|c|c|}
\hline \multirow{2}{*}{\multicolumn{1}{c|}{ Flight phase }} & \multicolumn{3}{c|}{ Control mode } \\
\cline { 2 - 4 } & manual & flight director & autopilot \\
\hline En Route Oceanic & $2 \mathrm{NM}$ & $0.5 \mathrm{NM}$ & $0.25 \mathrm{NM}$ \\
\hline En Route Domestic & $1 \mathrm{NM}$ & $0.5 \mathrm{NM}$ & $0.25 \mathrm{NM}$ \\
\hline Terminal & $1 \mathrm{NM}$ & $0.5 \mathrm{NM}$ & $0.25 \mathrm{NM}$ \\
\hline Approach & $0.5 \mathrm{NM}$ & $0.25 \mathrm{NM}$ & $0.125 \mathrm{NM}$ \\
\hline
\end{tabular}




\section{Accuracy of positioning by navigational aids}

Modern algorithms can use a pair of ground navigational aids for positioning simultaneously. In particular, at each point of airspace, there is a set of available navigational aids services, but only a pair of sensors can be used according to equipment list limitation. Selection of navigational aids is grounded on error analysis. The root-mean-square deviation of positioning error in the horizontal plane is used as the main characteristic of positioning accuracy.

The error of positioning in a horizontal plane for a pair of DME A and DME B by TOA method can be defined as follows (Eurocontrol, 2008):

$\sigma_{D M E / D M E}^{2}=\left(2 \sigma_{\text {sis }}^{2}+\sigma_{\text {air DME A }}^{2}+\sigma_{\text {air DME B }}^{2}\right) \sin ^{-2} \alpha_{A B}$,

where $\sigma_{\text {sis }}^{2}$ includes error introduced by radio waves propagation in space and transponder error; $\sigma_{\text {air DME A, }}^{2} \sigma_{\text {air DME }}^{2}$ ${ }_{B}$ are errors introduced by the airborne interrogator in range measurements to DME A and DME B, correspondingly; $\alpha_{\mathrm{AB}}$ is the inclusion angle between the directions to DMEs.

According to almost the same characteristics of DME, the value of $\sigma_{\text {sis }}$ cannot exceed 0.05 NM to DME equipment first installed after 1 January 1989 (ICAO, 2006). The theoretical maximum value of error introduced by the airborne interrogator $\left(\sigma_{\text {air }}\right)$ is a function of measured range (ICAO, 2008b):

$$
\sigma_{\text {air }}=\max \{0.085 \mathrm{NM} ; 0.125 \% R\} \text {. }
$$

where $R$ is a measured range by DME.

An error of positioning by AOA method for a pair of VOR equipment can be represented as follows (Ostroumov et al., 2018):

$$
\sigma_{V O R / V O R}^{2}=\sigma_{V O R}^{2}\left(d^{2}{ }_{V O R}+d^{2}{ }_{V O R B}\right) \sin ^{-2} \alpha_{A B},
$$

where $\sigma_{\mathrm{VOR}}$ is the root mean square errors of angle measurement of the VOR system in radians. Typical $\sigma_{\mathrm{VOR}}$ does not exceed $1^{\circ} ; d_{V O R A, B}$ are distances to VORs; $\alpha_{A B}$ is the inclusion angle between the directions to VORs.

Also, the same formula (3) can be applied for positioning by angles to Non-Directional Beacons measured by ADF.

In the VOR/DME positioning approach VOR and DME, placed in one navigation point, are used. Root mean square error of VOR/DME positioning in a horizontal plane is:

$$
\sigma_{V O R / D M E}^{2}=\sigma_{D M E}^{2}+D_{h}^{2} \sigma_{V O R}^{2},
$$

where $D_{h}$ is horizontal distance between aircraft and VOR/DME.
An error analysis of positioning by navigational aids indicates the dependence of root mean square errors from ground network configuration and geometrical location of navigational aids for different positioning approaches DME/DME, VOR/VOR, and VOR/DME.

\section{Performance analysis in terms of set theory}

The geometric configuration of navigational aids network makes performance vary in the airspace of the service area. Unfortunately, there are no possible ways to get an exact mathematical function, which would describe the distribution of positioning errors by coordinates analytically. The main approach to performance analysis is to consider airspace as a set of elementary particles, with an estimation of positioning accuracy for each of them. The positioning characteristics are considered constant inside of each particle. Compliance with the requirements for navigational specifications is also considered to be constant. The sets of elementary particles that meet the requirements of a particular navigation specification form availability area of certain navigational characteristic. An accuracy of availability area construction, in this case, is determined by the resolution or geometric dimensions of the elementary particle space. In the general case, the airspace that meets the requirements for accuracy can be represented as a set of points, centers of elementary particles. The geometry of the mutual location and the technical characteristics of the navigational aids network determine the volumes of airspace within which it is possible to use them for positioning and navigation. In particular, each of the positioning methods by pair of navigational aids will match the set of compliance with the navigational specification RNAV 1, RNAV 2, RNP 4, or RNAV 5, as shown in Table 3.

In the general case, each of the RNAV sets is determined by a certain number of $k$ subsets of RNAVi (Figure 1):

$$
R N A V i \subset R N A V, i=1, \ldots, k .
$$

The RNAV sets can be considered as binary relations between sets that define the coordinates of elementary particle centers in case of analysis for a given flight level. The elements of space are specified in a certain coordinate system, for example, in a geodesic using sets of the latitude

\begin{tabular}{|c|c|c|c|}
\hline Navigation specification & DME/DME & VOR/DME & VOR/VOR \\
\hline RNAV 1 & RNAV $1_{\text {DME/DME }}$ & RNAV1 $1_{\text {VOR/DME }}$ & $\mathrm{RNAV}_{\mathrm{VOR} / \mathrm{VOR}}$ \\
\hline RNAV 2 & RNAV2 $2_{\text {DME/DME }}$ & RNAV2 VOR/DME & RNAV2 ${ }_{\text {VOR/VOR }}$ \\
\hline RNP 4 & $\mathrm{RNP}_{\mathrm{DME} / \mathrm{DME}}$ & RNP4 $4_{\text {VOR/DME }}$ & RNP4 $4_{\text {VOR/VOR }}$ \\
\hline RNAV 5 & RNAV5 $5_{\mathrm{DME} / \mathrm{DME}}$ & RNAV5 VOR/DME & RNAV5 $5_{\text {VOR/VOR }}$ \\
\hline
\end{tabular}
$\Phi$, longitude $\Lambda$ and heights $\mathrm{H}$ :

$$
\begin{aligned}
& \Phi=\left\{\varphi \mid \varphi_{\text {min }} \leq \varphi \leq \varphi_{\text {max }}\right\}, \\
& \Lambda=\left\{\lambda \mid \lambda_{\text {min }} \leq \lambda \leq \lambda_{\text {max }}\right\},
\end{aligned}
$$

Table 3. Sets of compliance with navigational specifications 


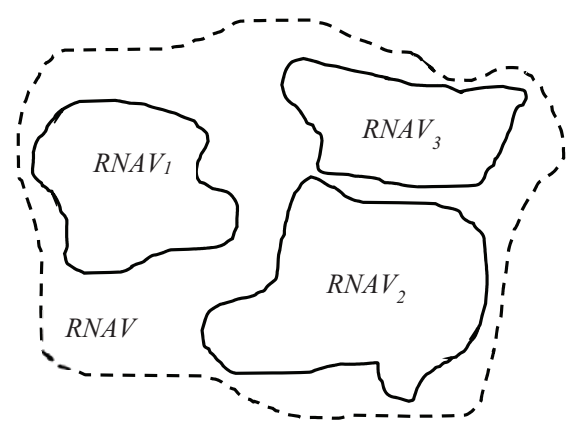

Figure 1. RNAVi subsets

$$
H=\left\{h \mid h_{\min } \leq h \leq h_{\max }\right\},
$$

where $\varphi_{\text {min }}, \varphi_{\max }, \lambda_{\text {min }}, \lambda_{\text {max }}, h_{\text {min }}, h_{\text {max }}$ are the boundary limits that determine the studied volume of airspace. Also, space elements can be specified in the Cartesian coordinate system, for example, in the local NEU using $X$, $Y, Z$ sets:

$$
\begin{aligned}
& X=\left\{x \mid x_{\text {min }} \leq x \leq x_{\text {max }}\right\}, \\
& Y=\left\{y \mid y_{\text {min }} \leq y \leq y_{\text {max }}\right\}, \\
& Z=\left\{z \mid z_{\text {min }} \leq z \leq z_{\text {max }}\right\},
\end{aligned}
$$

where $x_{\text {min }}, x_{\text {max }}, y_{\text {min }}, y_{\text {max }}, z_{\text {min }}, z_{\text {max }}$ are the boundaries defining the studied volume of airspace in the Cartesian coordinate system.

Any non-empty subset of $R N A V_{i}$ can be considered as ternary relations between sets that specify the coordinates of space elements:

$$
R N A V_{i} \subseteq X \times Y \times Z .
$$

In this case, the Cartesian product of the $X, Y, Z$ sets forms a set of ordered triples of coordinates $<\mathrm{x}, \mathrm{y}, \mathrm{z}>$ consisting of elements of these sets:

$$
X \times Y \times Z=\{\langle x, y, z>| x \in X, y \in Y, z \in Z\} .
$$

In partial case, for a fixed flight level a binary relation can be considered as follows:

$$
\begin{aligned}
& R N A V_{F L, i} \subseteq X \times Y, \\
& X \times Y=\{<x, y>\mid x \in X, y \in Y\} .
\end{aligned}
$$

In terms of assessing compliance with the characteristics of area navigation, it is important to evaluate the total number of compliance with the $R N A V$ requirements for each positioning methods by pairs and a set of navigational aids. That is, estimating the boundaries of the PBN set for which the $R N A V_{D M E / D M E}, R N A V_{V O R / D M E}, R N A V$ $V O R / V O R, R N A V_{D M E s}, R N A V_{V O R / D M E s}, R N A V_{V O R s}$ sets can be considered as subsets:

$$
\begin{aligned}
& R N A V_{D M E / D M E} \subset P B N ; R N A V_{V O R / D M E} \subset P B N ; \\
& R N A V_{V O R / V O R} \subset P B N ; R N A V_{D M E s} \subset P B N ; \\
& R N A V_{V O R / D M E s} \subset P B N ; R N A V_{V O R s} \subset P B N .
\end{aligned}
$$

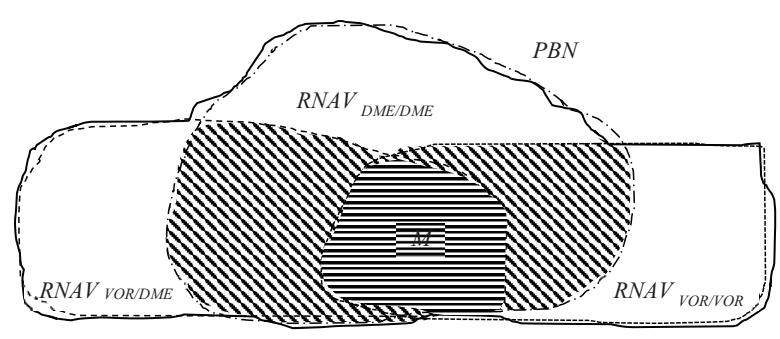

Figure 2. $P B N$ set

As shown in Figure 2 the PBN set forms a set of coordinates of the centers of the elementary particles within which the navigation of the aircraft is allowed in accordance with a particular specification. Thus, it is possible to write:

$$
\begin{aligned}
& P B N \subseteq R N A V_{D M E / D M E} \cup R N A V_{V O R / D M E} \cup R N A V_{V O R / V O R} \cup \\
& R N A V_{D M E s} \cup R N A V_{V O R / D M E s} \cup R N A V_{V O R s}
\end{aligned}
$$

The inverse $\overline{P B N}$ set corresponds to the area of the space within which navigation by navigational aids can not be provided:

$$
\overline{P B N}=\left\{L_{i} \mid L_{i} \not \subset P B N\right\} \text {. }
$$

The power of the PBN set can determine the airspace area or volume that satisfies certain navigation specification requirements. The area of compliance with a specific navigation specification for a given altitude may be calculated as follows:

$$
\begin{aligned}
& S_{F L, R N A V}=|P B N| S_{0}, \\
& S_{0}=\left(x_{\text {max }}-x_{\text {min }}\right)\left(y_{\text {max }}-y_{\text {min }}\right) n^{-2},
\end{aligned}
$$

where $S_{0}$ is the area of the elementary partcle of airspace; $n$ is a total number of elements. Substituting (8) into (7) and taking into account: $n=|X|=|Y|$, we have:

$$
S_{F L, R N A V}=\left(x_{\text {max }}-x_{\text {min }}\right)\left(y_{\text {max }}-y_{\text {min }}\right)|P B N||X|^{-2} \text {. }
$$

The volume of airspace meeting navigation specification requirements, with a cubic elementary particle, can be written as follows:

$$
\begin{aligned}
& V_{R N A V}=|P B N| V_{0}, \\
& V_{0}=\left(x_{\text {max }}-x_{\text {min }}\right)\left(y_{\text {max }}-y_{\text {min }}\right)\left(z_{\text {max }}-z_{\text {min }}\right) n^{-3} .
\end{aligned}
$$

Similar to (9), we have:

$$
V_{R N A V}=\left(x_{\max }-x_{\min }\right)\left(y_{\max }-y_{\min }\right)\left(z_{\max }-z_{\min }\right)|P B N||X|^{-3} \text {. }
$$

The redundancy of the navigation methods at each point of the space can be formalized due to the association of the RNAVi sets. The PBN series can be considered to be bound if the subsets of RNAV correspond to subsets of the cross section (Figure 2):

$$
\begin{aligned}
M= & R N A V_{D M E / D M E} \cap R N A V_{V O R / D M E} \cap R N A V_{V O R / V O R},(10) \\
& M \neq \varnothing .
\end{aligned}
$$

The number of associated subsets corresponds to the number of permitted methods of aircraft positioning at a 
certain point of airspace. Availability areas of only a particular positioning method can be defined as the difference in the sets of each of the methods:

$P B N_{D M E / D M E}=\left(R N A V_{D M E / D M E} \backslash R N A V_{V O R / D M E}\right) \backslash R N A V_{V O R / V O R}$,
$P B N_{V O R / D M E}=\left(R N A V_{V O R / D M E} \backslash R N A V_{D M E / D M E}\right) \backslash R N A V_{V O R / V O R}$,
$P B N_{V O R / V O R}=\left(R N A V_{V O R / V O R} \backslash R N A V_{V O R / D M E}\right) \backslash R N A V_{D M E / D M E}$.

The availability volumes study of different positioning methods allows analyzing the airspace with the purpose of ensuring the requirements of navigational specifications. In addition, airspace analysis in terms of set theory helps to identify areas of space (6) that do not meet PBN requirements and develop a strategy for improving air navigation services.

\section{Numerical demonstration}

We will conduct an analysis of Ukrainian airspace regarding compliance with the requirements of area navigation for positioning by navigational aids. The ground network of navigational aids consists of 12 DMEs (in particular: BAH, IHA, IHR, IKI, IKV, KSN, KVR, ILO, ILV, STB, VIN, YHT) and 8 VOR / DMEs (BRP, DNP, IVF, KHR, KVH, LIV, ODS, SLV) (AIP, 2017) represented by high altitude and terminal types (Federal Aviation Administrations [FAA], 1982). For each type of navigational aid, there is a certain area of airspace within which the services of the navigational aid are guaranteed. To reduce the computational time, we use a cylindrical model of navigational aids service volume limited above by a cone of silence (FAA, 2007). An investigated airspace has been divided into 200 squared elementary particles in the East-West direction and 107 particles to cover investigated area in the NorthSouth direction. The area of each elementary particle is $75 \mathrm{~km}^{2}$ that indicates a resolution of the analysis. Thus, a matrix of investigated airspace includes 21400 elementary particles. There are 11,257 elementary particles within the boundaries of the investigated airspace, which will cover 852 thousand $\mathrm{km}^{2}$ approximately. A number of elementary particles defines the precision of analysis but is limited by the performance of computation equipment. The results of the availability assessment within Ukrainian airspace at FL 195 taking into account national navigational aids networks of the neighboring countries: Poland, Slovakia, Hungary, Romania, Moldova, Russia, Belarus, and Turkey are shown in Figure 3.

The subsets of the all available navigational aids at each elementary particle of airspace is used for forming pairs and estimating the NSE by (2), (3), (4) separately for each positioning method. The navigational aids pair, which provides the minimum NSE value, is considered as an optimal one. The choice of the optimal pair is performed by Binary Integer Linear Programming (Ostroumov et al., 2018). TSE value is calculated by (1), taking the assumption of FTE $=0.5 \mathrm{NM}$ for flight director control mode according to Table 2. The TSE is used as a criterion for compliance with the requirements of RNAV according to Table 1 . Figures 4, 5, 6 show the results of estimating the sets of compliance with RNP/RNAV requirements for DME/DME, VOR/DME and VOR/VOR positioning methods, respectively.

The navigational specifications availability areas allow analyzing geographically the disadvantages of the navigational aids network geometry. In particular, the RNAV 2 areas for VOR/VOR are provided mostly only between the arcs connecting BRP and $\mathrm{KVH}$, and RNAV

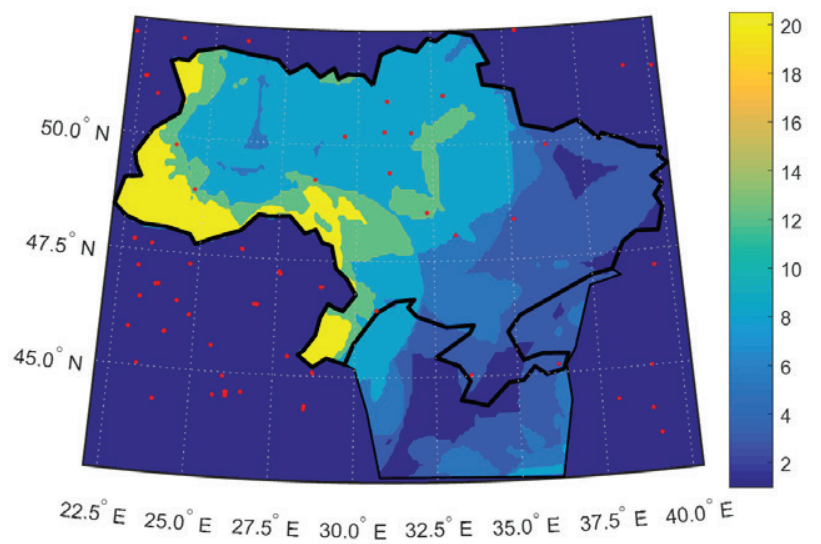

Figure 3. Amount of available navigational aids in Ukrainian airspace

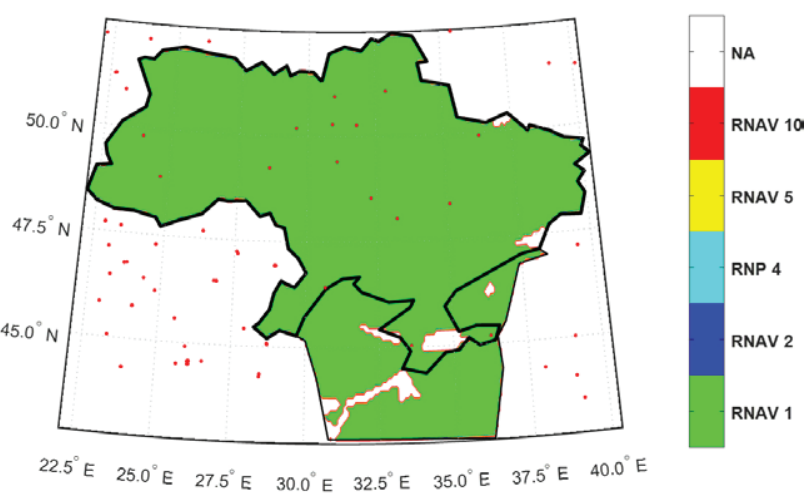

Figure 4. Compliance of positioning by DME/DME with RNP/RNAV

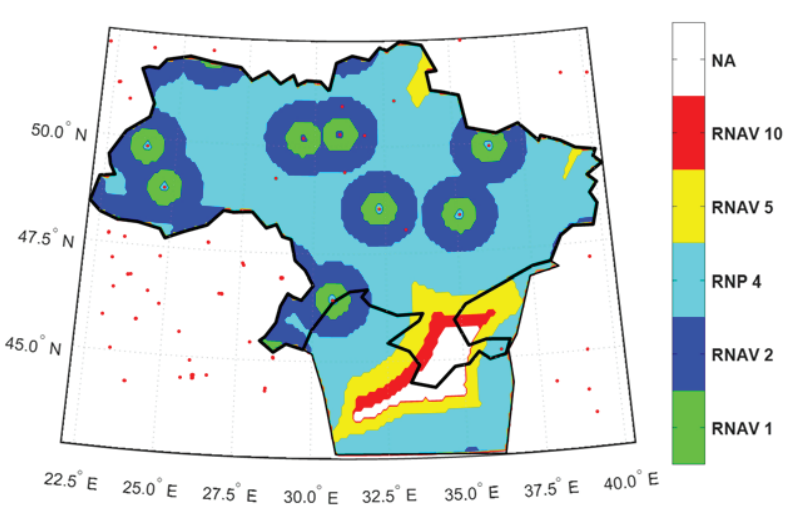

Figure 5. Compliance of positioning by VOR/DME with RNP/RNAV 


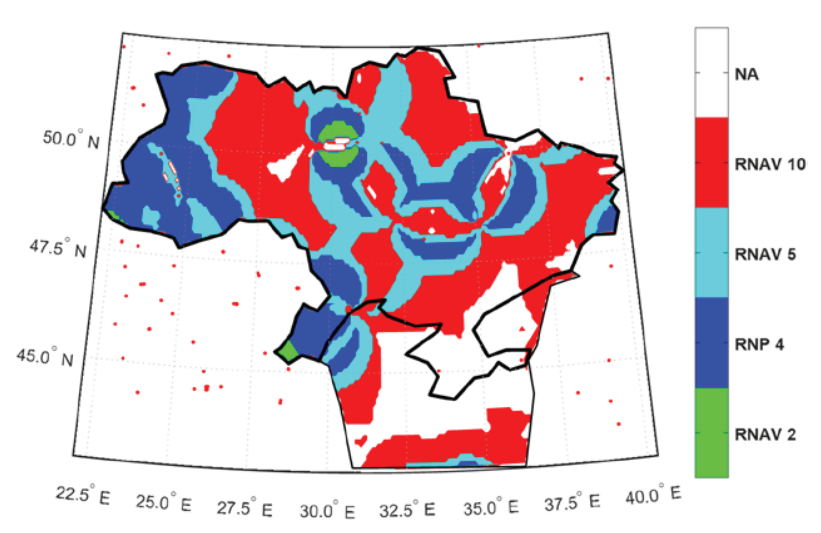

Figure 6. Compliance of positioning by VOR/VOR with RNP/RNAV

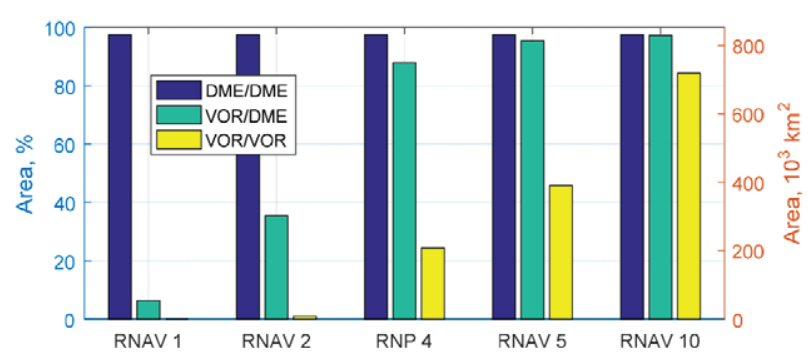

Figure 7. An area analysis of navigation specifications compliance for FL195

1 requirements are not provided at all, due to errors of angular data provided by VOR equipment. In general, any of the navigational characteristics are not supported by the AOA method at the Eastern and Southern airspace. The AOA/TOA method provides positioning in accordance with the requirements of RNAV 1 only in a circular area in the immediate vicinity of the ground equipment, although the requirements of RNP 4 and RNAV 5 are performed in the multiple parts of airspace except for the area above the Crimea. Positioning by DME/DME is met RNAV 1 requirements and higher in the almost of investigated volume. The results of area analysis for a variety of navigation specifications according to different navigation methods by (9) are given in Figure 7.

About $97.4 \%$ of investigated airspace at FL195 meets the RNAV requirements for DME/DME according to Figure 7. The VOR/DME and VOR/VOR indicate a tendency to increase, depending on the reduction of the RNAV precision requirements.

In addition, the ability of airspace to support various positioning methods define continuity characteristic. Results of intersection area study by (10) of DME/DME and VOR/DME positioning methods in RNAV airspace in comparison with all available methods are indicated in Figure 8.

Also, the histogram in Figure 8 reflects the ability of the investigated airspace to provide services of a backup navigation system in case of a minor failure with one of both DME or VOR sensors onboard.

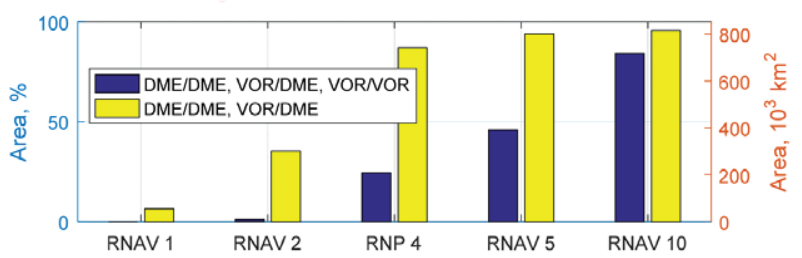

Figure 8. An area supporting various positioning methods simultaneously

\section{Conclusions}

The proposed approach to the evaluation of the characteristics of the aircraft onboard navigation system allows a geometrical analysis of the compliance areas with certain navigational specifications and to allocate areas of unavailability of positioning by particular methods. Geometric analysis of areas allows developing steps to improve the quality of air navigation services provided by ANSP and improving air traffic safety. Investigation of the sets of compliance with certain navigational specifications allows estimating the general characteristics of airspace compliance with RNAV requirements. The analysis of intersections between RNAV sets allows studying the ability of air navigation services to ensure the continuity of navigation information in case of on-board sensors failure.

The detailed analysis of air navigation service according to RNAV requirements within Ukrainian airspace allows concluding the quality of positioning services by ground navigational aids. The obtained results are important for roadmap development to improve the safety of air transportation and are the necessary inputs to assess the risks of dangerous deviations and aircraft collision.

\section{References}

AIP (2017). Aeronautical Information Publication of Ukraine. Ukrainian State Air Traffic Services Enterprise.

Dabbakuti, J. R., Ratnam, D. V., \& Sunda, S. (2016). Modeling of ionospheric time delays based on adjusted spherical harmonic analysis. Aviation, 20(1), 1-7.

https://doi.org/10.3846/16487788.2016.1162197

International Civil Aviation Organization. (2006). Aeronautical telecommunications. Radio navigation aids (Annex 10, Vol. 1). International Standards and Recommended Practices. International Civil Aviation Organization, Montreal, Canada.

International Civil Aviation Organization. (2008a). Unified framework for collision risk modeling in support of the manual on airspace planning methodology with further applications, (CIR 319, AN/181 ed.). International Civil Aviation Organization, Montreal, Canada.

International Civil Aviation Organization. (2008b). Performancebased navigation (PBN) manual (Doc 9613 AN/937). International Civil Aviation Organisation, Montreal, Canada.

International Civil Aviation Organization. (2012). Global Navigation Satellite System (GNSS) manual (Doc 9849, AN/457). International Civil Aviation Organization, Montreal, Canada.

Eurocontrol. (2008). Guidelines for PRNAV Infrastructure Assessment (GUID-114, 1.2 ed.). 
Federal Aviation Administrations. (1982). AC 00-31A - U.S National aviation standard for very high frequency omnidirectional radio range (VOR)/ distance measuring equipment (DME)/ tactical air navigation (TACAN) systems. 67.

Federal Aviation Administrations. (2007). AC 90-100A - U.S Terminal and En Route Area Navigation (RNAV) Operations. 273.

Han, S., Gong, Z., Meng, W., Li, C., \& Gu, X. (2016). Future alternative positioning, navigation, and timing techniques: A survey. IEEE Wireless Communications, 23(6), 154-160. https://doi.org/10.1109/MWC.2016.1500181RP

Kasperovych, N., Shvets, V., \& Ostrovsky, Y. (2008). Noise modeling for global satellite aeronavigation systems. In 2008 Proceedings of Microwaves, Radar and Remote Sensing Symposium, MRRS 2008, (pp. 310-313). IEEE. https://doi.org/10.1109/MRRS.2008.4669602

Kutsenko, O., Ilnytska, S., \& Konin, V. (2018). Investigation of the residual tropospheric error influence on the coordinate determination accuracy in a satellite landing system. Aviation, 22(4), 156-165. https://doi.org/10.3846/aviation.2018.7082

Kuzmenko, N. S., Ostroumov, I. V., \& Marais, K. (2018). An accuracy and availability estimation of aircraft positioning by navigational aids. In 2018 Proceedings of Methods and Systems of Navigation and Motion Control, MSNMC 2008, (pp. 36-40). IEEE. https://doi.org/10.1109/MSNMC.2018.8576276

Lo, S., Enge, P., Niles, F., Loh, R., Eldredge, L., \& Narins, M. (2010). Preliminary assessment of alternative navigation means for civil aviation. International Technical Meeting 2010, ITM 2010, 1, 484-492.

Lubbers, B., Mildner, S., Onincx, P., \& Scheele, A. (2015). A study on the accuracy of GPS positioning during jamming. In 2015 International Association of Institutes of Navigation World Congress, IAIN 2015 - Proceedings. IEEE.

https://doi.org/10.1109/IAIN.2015.7352258

Muller, D., Uday, P., \& Marais, K. B. (2011). Evaluation of the potential environmental benefits of RNAV/RNP arrival procedures. Paper presented at the 11th AIAA Aviation Technology, Integration, and Operations (ATIO) Conference, Including the AIAA Balloon Systems Conference and 19th AIAA Lighterthan-Air Technology Conference. https://doi.org/10.2514/6.2011-6932

Narins, M., Eldredge, L., Enge, P., Harrison, M., Kenagy, R., \& Lo, S. (2012, April). Alternative position, navigation, and timing - The need for robust radionavigation. In Global Navigation Satellite Systems: Report of a Joint Workshop of the National Academy of Engineering and the Chinese Academy of Engineering (pp. 119-136). The National Academies Press.

Ostroumov, I. V., Kuzmenko, N. S., \& Marais, K. (2018). Optimal pair of navigational aids selection. In 2018 Proceedings of Methods and Systems of Navigation and Motion Control, MSNMC 2018 (pp. 32-35). IEEE. https://doi.org/10.1109/MSNMC.2018.8576293

Vitan, V., Berz, G., Saini, L., Arethens, J., Belabbas, B., \& Hotmar, P. (2018). Research on alternative positioning navigation and timing in Europe. In ICNS 2018 - Integrated Communications, Navigation, Surveillance Conference, (pp. 4D21-4D217). IEEE. https://doi.org/10.1109/ICNSURV.2018.8384887

\section{Appendix}

\section{Abbreviations}

ADF - Automatic Direction Finder;

ANSP - Air Navigation Service Provider;

AOA - Angle of Arrival;

DME - Distance Measuring Equipment;

FL - Flight Level

FTE - Flight Technical Error;

GNSS - Global Navigation Satellite System

NSE - Navigation System Error;

PBN - Performance-based Navigation;

PDE - Path Definition Error.

RNAV - Area navigation;

RNP - Required Navigation Performance;

SESAR - Single European Sky ATM Research;

TOA - Time of Arrival;

TSE - Total System Error;

VOR - VHF omnidirectional range. 